\title{
Simulations of the Atmospheric Boundary Layer in a Wind Tunnel with Short Test Section
}

\author{
Luciana Bassi Marinho Pires ${ }^{1}$, Igor Braga de Paula², Gilberto Fisch ${ }^{3}$, Ralf Gielow², \\ Roberto da Mota Girardi ${ }^{4}$
}

\begin{abstract}
This article presents a study of three different passive devices (spires, screens, and a carpet) separately and in various combinations, to simulate the atmospheric boundary layer $(A B L)$ in a wind tunnel with a short test chamber $(465 \times 465 \times 1200 \mathrm{~mm})$. The influence of distances between these devices on the formation of the $A B L$ is established, and optimization of variation of thicknesses of the screens (thin, medium, and coarse) on pressure loss is explored. The results obtained in this work gave support for the analysis of the atmospheric flow and turbulence at Alcantara Launching Center (ALC) in order to launch Brazilian space vehicles under safe conditions. The results show that the "spires" and the thin screen are the devices that require the least area to form an $\mathrm{ABL}$ in a test chamber. The physical proximity of two devices (the spires and the medium screen) also influences the size of the $A B L$ formed, which varies from 180 to $200 \mathrm{~mm}$. The power law exponent ranged from 0.12 up to 0.14 after the insertion of a carpet.
\end{abstract}

KEYWORDS: Passive methods, Spires, Carpet, Screens, Wind power law.

\section{INTRODUCTION}

The first boundary layer (BL) wind tunnel was built in 1965 by Davenport (1967) at Western University (formerly University of Western Ontario), Ontario, Canada. The studies of Jensen and Franck $(1963,1965)$ and Franck $(1963)$ in wind tunnels concluded that it would be possible to obtain an appropriate scale of the natural wind structure by covering a considerable length of the wind tunnel's floor with a material of suitable roughness. However, the disadvantage of this process is that it requires a length of about $25 \mathrm{~m}$ to form a BL with 60 to $120 \mathrm{~cm}$ height, which is possible only in tunnels with a long test chamber (Blessmann, 1973). Thus, improved techniques for reproduction of the main characteristics of natural winds, as well as the formation of the atmospheric boundary layer $(\mathrm{ABL})$, are needed. These techniques will permit shorter test chambers, so that existing aeronautical tunnels could be used for atmospheric simulations of meteorological interest, with the advantage of flow control and improved data collection. The objective of the current article is to provide directions to fulfill the challenges of adaptation of aeronautic wind tunnels for meteorology purposes, cataloging the steps of modification and their expected results. This work was done at Departamento de Ciência e Tecnologia Aeroespacial (DCTA) wind tunnel to give support for the analysis of the atmospheric flow and turbulence at Alcantara Launching

1.Instituto Nacional de Pesquisas Espaciais - São José dos Campos/SP - Brazil 2.Pontifícia Universidade Católica do Rio de Janeiro - Rio de Janeiro/RJ - Brazil 3. Instituto de Aeronáutica e Espaço - São José dos Campos/SP - Brazil 4.Instituto Tecnológico de Aeronáutica - São José dos Campos/SP - Brazil Author for correspondence: Luciana Bassi Marinho Pires | Instituto Nacional de Pesquisas Espaciais - INPE/CPTEC | Avenida dos Astronautas, 1.758 Jardim da Granja | CEP 12.227-010 São José dos Campos/SP - Brazil | Email: lubassimp@gmail.com

Received: 13/11/12 | Accepted: 15/05/13 
Center (ALC). This is an ongoing project that aims to develop technical tools for Aerospace Meteorology associated with the launching of the Satellite Vehicle Launcher (VLS) within the Brazilian Space Program (Pires et al., 2010).

There are several studies using the wind tunnel for simulating the characteristics and behavior of the atmosphere. Some examples are the works of Novak et al., (2000) analyzing the turbulent structure of the atmosphere within and above canopies; Kwon et al., (2003) simulating the atmospheric wind field over a complex topography in order to plan the Naro Space Center in South Korea; Mavroidis et al., (2003), studying pollutant dispersion fields immersed in obstacles; Cao and Tamura (2006), simulating the turbulent BL flow over 2D steep, smooth, and rough hills in Tokyo. Kozmar (2009) reproduced the BL at different scales inside the wind tunnel to address the proper choice of simulation length scale and concluded that the length-scale factor does not influence the generated ABL models when using similarity criteria; and recently, Carpentieri et al., (2012) worked to quantify the mean and turbulent flow in geometries of real street canyon intersections, focusing on the area surrounding the intersection between Marylebone Road and Gloucester Place in Central London, UK. Recently, Avelar et al., (2012) did a detailed analysis of the atmospheric flow at ALC using a large wind tunnel. They have constructed the ABL profile using a combination of barrier and small blocks of wood. As all of them used wind tunnels for their experiments, new techniques and methods to develop the $\mathrm{ABL}$ are necessary in order to use different sizes of wind tunnel section tests.

The methods of simulating the ABL in wind tunnels are divided into passive and active types. The passive methods use barriers such as grilles, flat plates, triangular plates, writing pads, spires, carpets, etc. The active methods are those that use air jets to form a wall of fluid. The objective of this work is to present some of the passive devices (screens, spires, and carpet) used for the formation of the BL in wind tunnels. It will be done analyzing the optimizations of the formation of the ABL with the use of such devices as a function of both the distance among them and their characteristics (grids with larger or smaller spacing) based primarily on the mean velocity profile and turbulence intensity profiles. The main focus was on rural terrain. However, additional studies could investigate whether this approach works well when applied to other types of terrains (e.g., suburban, urban) with different geometries.

\section{MATERIAL AND METHODS}

This work was carried out in the commercially available open subsonic wind tunnel Plinth \& Partners LDD Wokingham Berkshire England (Serial No. 44/5065 TE) at the Kwein Lien Feng Laboratory at Instituto Tecnológico de Aeronáutica (ITA)/ DCTA, São José dos Campos, SP, Brazil (Fig. 1a). Its test section is square with cut corners $(465 \times 465 \mathrm{~mm})$ with a length of $1200 \mathrm{~mm}$. For this study, a channel apparatus with a width of $410 \mathrm{~mm}$ and length of $1200 \mathrm{~mm}$, consisting of an uncovered wooden frame, with free edges and side walls parallel to each other and perpendicular to the floor of the wind tunnel, was used to extend the test section for the formation of the ABL. The walls of the tunnel and of the "channel" (Fig. 1b) do not coincide, to minimize the lateral BL of the tunnel. An automatic positioner device with an arm capable of moving in three directions perpendicular to each other and with an accuracy of tenths of a millimeter was used, coupled to a computer, with the data collection points inserted by automatic handling equipment or manually (Fig. 1b). The positioner consists of the following elements: (i) positioner, ("traversing") from Dantec Dynamics serial code 9057 h 0123, Denmark, (ii) controls for the positioner, Dantec type $57 \mathrm{~b} 100$; and (iii) command manual positioner, serial code $9055 X 530$. The same configuration was utilized by Roballo (2007). The atmospheric flow is simulated by $22 \mathrm{~kW}(30 \mathrm{hp}$ ) electric fans for the range of the windspeeds. The maximum windspeed was $33 \mathrm{~m} / \mathrm{s}(120 \mathrm{~km} / \mathrm{h})$.

For initial adjustments, passive devices - screen and spires - were used and for fine tuning a wrinkled carpet was added. It is important to note that the insertion of screens within wind tunnels is usually done with a primary objective of reduction of turbulence of flow, because they have a tendency to uniform the flow and to break the big vortices into smaller ones that decay quickly (energy cascade process). However, in this study, the screens were used to accelerate the formation of the ABL in the test section.

Three types of screens with different meshes were used: (i) nylon square mesh with spacing of $2 \times 2 \mathrm{~mm}^{2}$ and $0.4 \mathrm{~mm}$ in diameter, called "thin screen," (ii) metal square mesh with spacing of $5.5 \times 5.5 \mathrm{~mm}^{2}$ and $1.0 \mathrm{~mm}$ diameter, called "medium screen"; and (iii) metal mesh type "beehive" with a spacing of $19.0 \times 17.0 \mathrm{~mm}^{2}$ and $0.5 \mathrm{~mm}$ in diameter, called "coarse screen" (Fig. 2). All these screens were available in local shops. The screens were assembled in a rigid frame made of steel stripes 


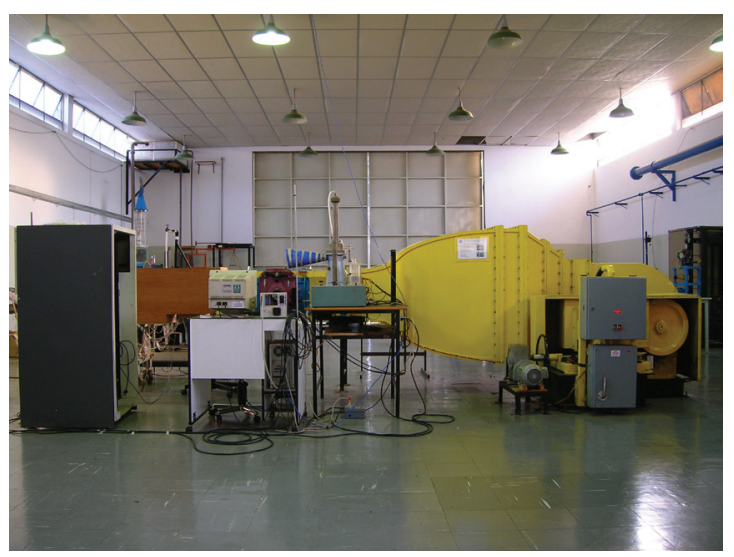

(a)

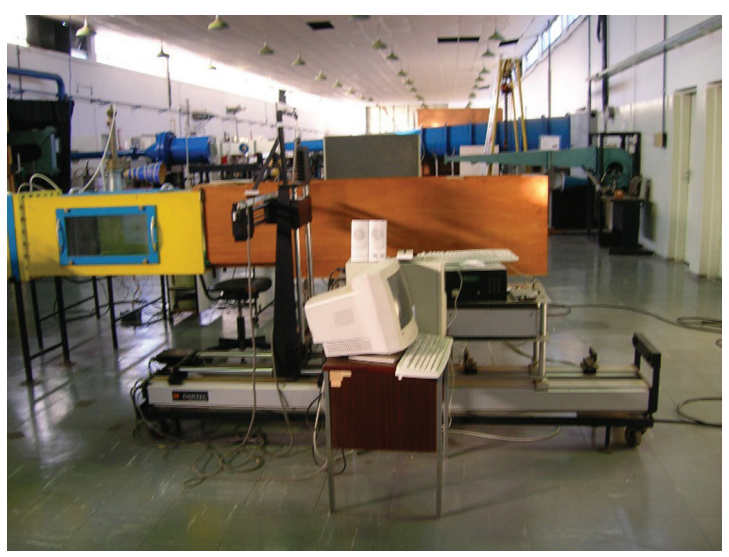

(b)

Figure 1. Wind tunnel of Prof. Kwein Lieng Feng Laboratory (ITA) (a) and automatic positioner and extension attached (b).

of $2 \mathrm{~mm}$ thick. Their tension was adjusted with small plates used to tighten the mesh to the frame. This was necessary in order to avoid the screen bending from aerodynamic loads during measurements. The mechanism created a small step of approximately $3 \mathrm{~mm}$ (approximately 1\% of the BL thickness) at the basis of the BL. This height is considered small for the current experiments. For instance, the carpet used to form the ABL had nearly the same height as the step, but a much longer length. Thus, the influence of such a small step on the ABL was assumed to be negligible.

The spires consist of triangular plates arranged in the test chamber entrance which, combined with the surface roughness, are used to generate the windspeed profile of the BL. The dimensions of the spires depend on the desired $\mathrm{BL}$ and the dimensions of the tunnel. It is recommended that the distance between two spires should be half of the height of one spire, and their heights should be smaller than the height of the wind tunnel (Pires, 2009). It is assumed, according with Blessman (1973), that the BL real $\delta$ is around $280 \mathrm{~m}$. The tunnel test chamber used has a height of $460 \mathrm{~mm}$ and the reduction of scale with respect to the actual size was 1:1000. Therefore it should produce a ABL as close as possible to $280 \mathrm{~mm}$, which was also checked by numerical simulations

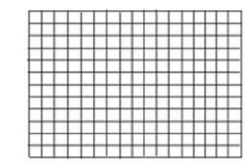

Thin screen

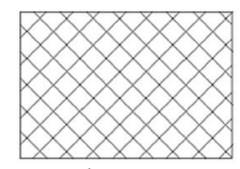

Medium screen

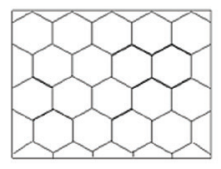

Coarse screen
Figure 2. Screens used for the formation of atmospheric boundary layer.
(Pires, 2009). Details regarding the calculation of the quantity and characteristics of spires that need to be used for different wind tunnel dimensions can be found in Blessmann (1973) and Pires (2009). For this work, three spires, each with heights of $307.7 \mathrm{~mm}$ and base width of $32.6 \mathrm{~mm}$, were used. The spires (Irwin, 1981) were produced using steel plates, with each spire consisting of two plates cut and folded together by spot welding (Fig. 3).

Hot-wire measurements were performed with a singlewire BL probe ("Dantec 55P15") of the constant-temperature hot-wire anemometer ("DANTEC Streamline bridge"). The calibration of the probe was performed using a DANTEC calibration unit. The equipment enables a precise adjustment of flow velocity at the exit of a nozzle. The dynamic pressure of the jet was measured with a standard Betz type manometer, and the respective velocities were correlated with

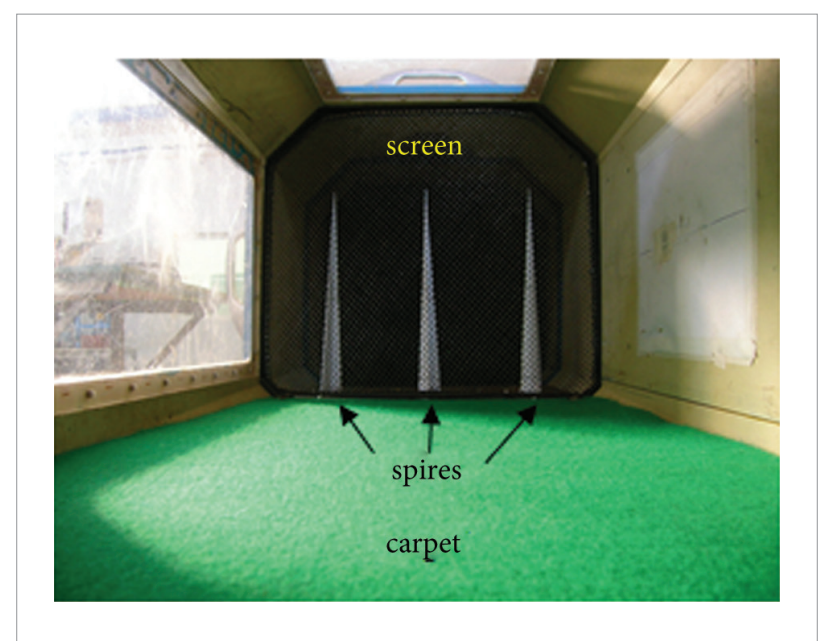

Figure 3. Devices used: carpet, screen, and spires. 
the hot-wire signal by the King's law. The procedure resulted in calibration errors below $0.5 \%$, which are typical of hot-wire applications. During the measurements, the hot-wire signal was acquired with an AD card (NI-PCI-MIO-16) with a resolution of 16 bits. To avoid clipping effects, the gain applied to analog signal was fixed; this kept the maximum voltage always below $80 \%$ of the range of the AD card. The DC and AC parts of the signal were not split prior to the acquisition; therefore no high-pass filtering was applied. Only low-pass filters were used to avoid aliasing effects. The positioning of the probe was done with a DANTEC traverse mechanism, which provides a resolution of $6.25 \mu \mathrm{m}$ in all three axes.
One important issue of $\mathrm{BL}$ measurements is the definition of the surface location. In the current experiments, the initial position was set by moving slowly the probe toward the wall until it visually touched the surface. Afterward, a correction based on the extrapolation of the mean flow data was applied. The procedure resulted in a nearly constant shift of approximately $1 \mathrm{~mm}$. The experimental apparatus is shown in Figs. 4 and 5. It uses an $x$ (longitudinal), $y$ (lateral), and $\mathrm{z}$ (vertical) coordinate system to describe the positions of measurements. Hot-wire anemometers of constant temperature were used to measure the flow. These anemometers utilize a several millimeter long tungsten

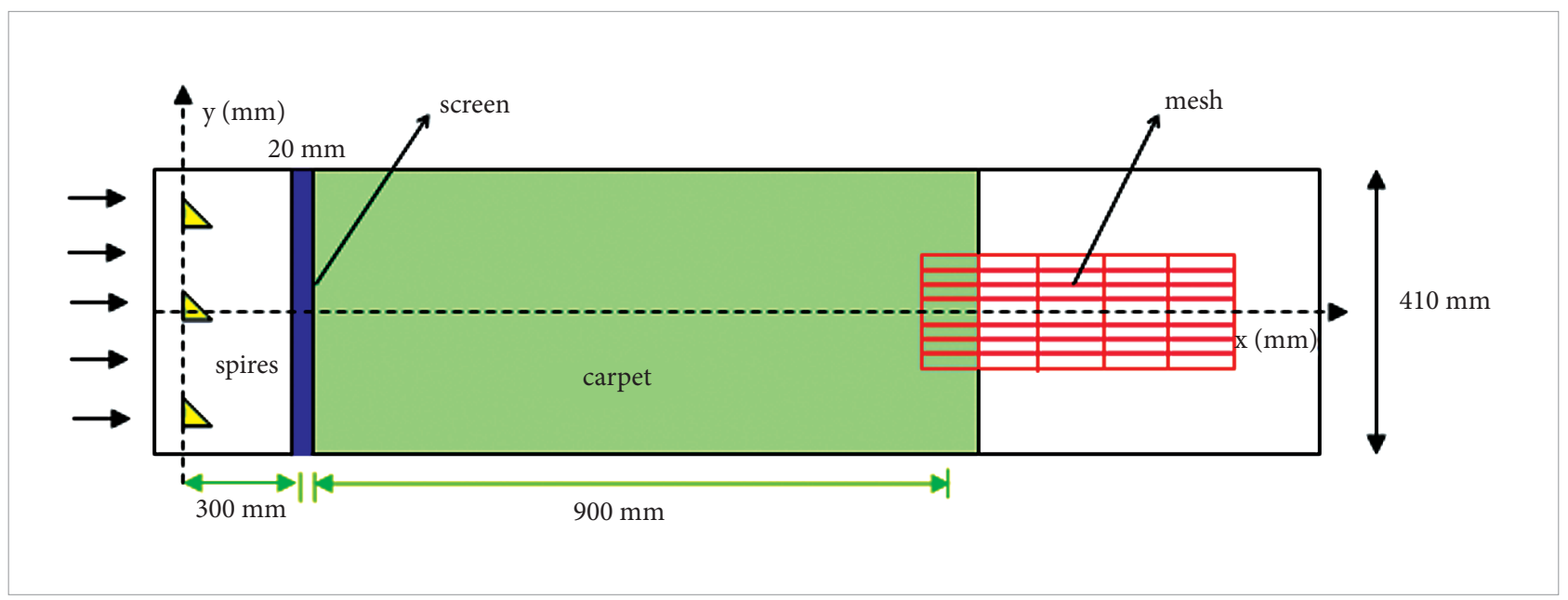

Figure 4. View of the arrangement of the experimental apparatus with the $x$ (longitudinal) and y (lateral) coordinates.

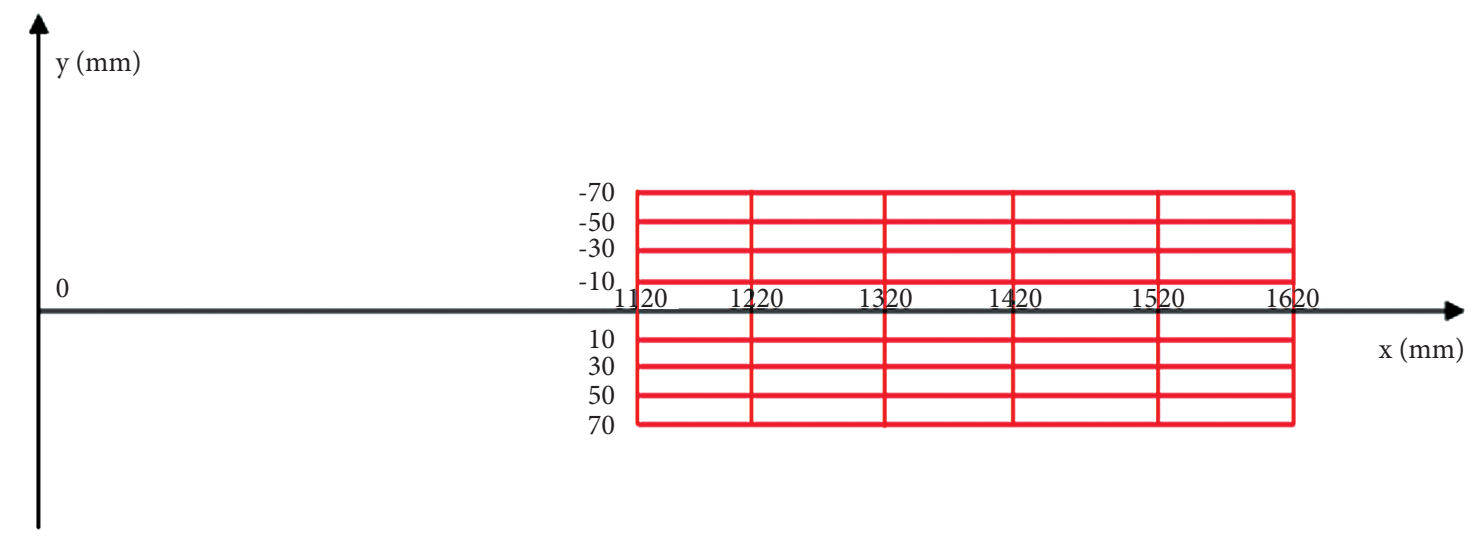

Figure 5. View of the mesh where measurements were taken. 
wire with a diameter of around $4 \mu \mathrm{m}$. The system functions as one of the resistors in a Wheatstone bridge, allowing measurements with high spatial and temporal resolutions. Further details about this technique can be seen in Roballo (2007). For data acquisition, we used the Labview computer program. The positions on the longitudinal and lateral axes are shown in Fig. 5. The vertical profile was performed at the heights $1,3,6,10,20,30,40,50,60,80,100,130,160,190$, 220 , and $260 \mathrm{~mm}$, using a scale factor of 1:1000.

The experimental uncertainty in the measurement of pressure and temperature is 0.1 and $0.4 \%$ respectively. This result is an uncertainty of $3.1 \%$ in the estimation of the Reynolds number, with the confidence interval of $95 \%$. More details can be found in Roballo (2007).

Initially, the spires and coarse, medium, and fine screen devices were tested separately.

Afterward, combinations of these devices were tested. Then, an optimization of the positions of spires and screen was done with distances of 150 and $300 \mathrm{~mm}$ from the screen. Finally, the carpet was inserted to perform the fine tuning of the desired roughness. The metric used to optimize the simulation of rural land was by the coefficient $\alpha$ of the wind power law, which was assumed equal to 0.15 .

\section{RESULTS AND DISCUSSION}

Table 1 presents the values of the coefficient $\alpha$ of the power law versus the Reynolds number $(\mathrm{Re})$ for the four devices (spires and fine, medium, and coarse screens) individually deployed, corresponding to the positions $\mathrm{x}=1120 \mathrm{~mm}$ and $\mathrm{x}=1620 \mathrm{~mm}$ grid points. Re was calculated for an ABL height of $280 \mathrm{~mm}$ as suggested by Pires et al., (2009), and the windspeed used was $\mathrm{u}_{\infty}$. The largest pressure loss occurred for the fine screen, with a dynamic pressure of about $28.8 \mathrm{~mm} \mathrm{H}_{2} \mathrm{O}$ and a Re of $3.81 \times 10^{5}$. The pressure losses measured by the Pitot tube were smaller for the coarse screen $(\mathrm{v}=27.6 \mathrm{~m} / \mathrm{s})$. The spires presented the second highest pressure loss with Re equal to $4.16 \times 10^{5}$, which is not very relevant, since it will be coupled to another device for the generation of the ABL. The ideal configuration causes the smallest pressure loss and generates the $\mathrm{ABL}$ in the shortest possible distance of the test section. Note that the coefficient $\alpha$ is independent of Re or the longitudinal position ( $\mathrm{x}$ ) and is always smaller than 0.1 (ranging from 0.05 up to 0.09 ). This small value of $\alpha$ is due to absence of other passive devices to fine tune the generation of $A B L$.

The ABL was fully developed at $\mathrm{x}=1120 \mathrm{~mm}$, showing nonsignificant differences with the combination of spires and thin screen, due to different gaps between the spires and the screen $(150 \mathrm{~mm}$ or $300 \mathrm{~mm})$. The combination of the spires with the medium screen moved the ABL to the position $\mathrm{x}=1420 \mathrm{~mm}$, with the highest mean square deviation for spires $300 \mathrm{~mm}$ distant from the screen reaching $3.0 \mathrm{~m} / \mathrm{s}$ near the surface and $0.5 \mathrm{~m} / \mathrm{s}$ at the height of $260 \mathrm{~mm}$. For the combination of the spires with coarse screen, the ABL was not developed inside the test chamber section, presenting an irregular windspeed profile, requiring a longer test section for its formation. Table 2 summarizes the values of $\alpha$ and Re for spires combined with the fine and medium screens. The higher values of Re, of about $4.8 \times 10^{5}$, were obtained for the medium screen placed further away $(300 \mathrm{~mm})$ from the spires. Spires with the thin screen display lower values of Re $\left(3.9 \times 10^{5}\right)$ for both small and greater separations. The largest coefficient $\alpha$ occurs for spires close $(150 \mathrm{~mm})$ to medium screens, with values around 0.10 .

Table 1. Re and $\alpha$ for isolated spires or screens.

\begin{tabular}{|c|c|c|c|c|c|}
\hline Devices & Positions [mm] & Windspeed $[\mathrm{m} / \mathrm{s}]$ & Dynamic pressure ( $\mathrm{mm} \mathrm{H}_{2}$ ) & $\alpha$ & $\mathrm{Re}$ \\
\hline \multirow{2}{*}{ Spires } & $\mathrm{x}=1220$ & 24 & 32.8 & 0.06 & $4.16 \times 10^{5}$ \\
\hline & $x=1620$ & 24 & 32.8 & 0.09 & $4.16 \times 10^{5}$ \\
\hline \multirow{2}{*}{ Thin screen } & $\mathrm{x}=1220$ & 22 & 28.8 & 0.02 & $3.81 \times 10^{5}$ \\
\hline & $x=1620$ & 22 & 28.8 & 0.07 & $3.81 \times 10^{5}$ \\
\hline \multirow{2}{*}{ Medium screen } & $x=1220$ & 27 & 41 & 0.07 & $4.68 \times 10^{5}$ \\
\hline & $x=1620$ & 27 & 41 & 0.04 & $4.68 \times 10^{5}$ \\
\hline \multirow{2}{*}{ Coarse screen } & $\mathrm{x}=1220$ & 27.6 & 44 & 0.06 & $4.78 \times 10^{5}$ \\
\hline & $\mathrm{x}=1620$ & 27.6 & 44 & 0.05 & $4.78 \times 10^{5}$ \\
\hline
\end{tabular}


Table 2. Values of Re and $\alpha$ for spires combined with fine and medium screens.

\begin{tabular}{|c|c|c|c|c|}
\hline Devices & Positions [mm] & Windspeed $(\mathrm{m} / \mathrm{s})$ & $\alpha$ & $\mathrm{Re}$ \\
\hline \multirow{2}{*}{ Spires+thin screen } & $\mathrm{x}=1120$ & 22.5 & 0.03 & $3.9 \times 10^{5}$ \\
\hline & $\mathrm{x}=1220$ & 22.5 & 0.03 & $3.9 \times 10^{5}$ \\
\hline \multirow{3}{*}{ Spires+medium screen } & $x=1320$ & 28 & 0.06 & $4.8 \times 10^{5}$ \\
\hline & $\mathrm{x}=1420$ & 28 & 0.05 & $4.8 \times 10^{5}$ \\
\hline & $x=1520$ & 28 & 0.04 & $4.8 \times 10^{5}$ \\
\hline \multirow{2}{*}{ Spires close+thin screen } & $\mathrm{x}=1120$ & 23 & 0.08 & $3.9 \times 10^{5}$ \\
\hline & $\mathrm{x}=1220$ & 23 & 0.07 & $3.9 \times 10^{5}$ \\
\hline \multirow{3}{*}{ Spires close + medium screen } & $\mathrm{x}=1320$ & 24 & 0.11 & $4.2 \times 10^{5}$ \\
\hline & $\mathrm{x}=1420$ & 24 & 0.11 & $4.2 \times 10^{5}$ \\
\hline & $x=1520$ & 24 & 0.10 & $4.2 \times 10^{5}$ \\
\hline
\end{tabular}

Gray: position where the ABL is formed.

Measurements were carried out at several locations along the stream and spanwise directions, as shown schematically in Fig. 5. The variations of mean and turbulent flow profiles along the spanwise direction were used as a criterion to ensure that the
ABL was developed. Figure 6 shows an example of developed BLs, measured with medium screens. No significant variation of the profiles is present in the results, suggesting the presence of a welldeveloped flow at position $\mathrm{x}=1420$.

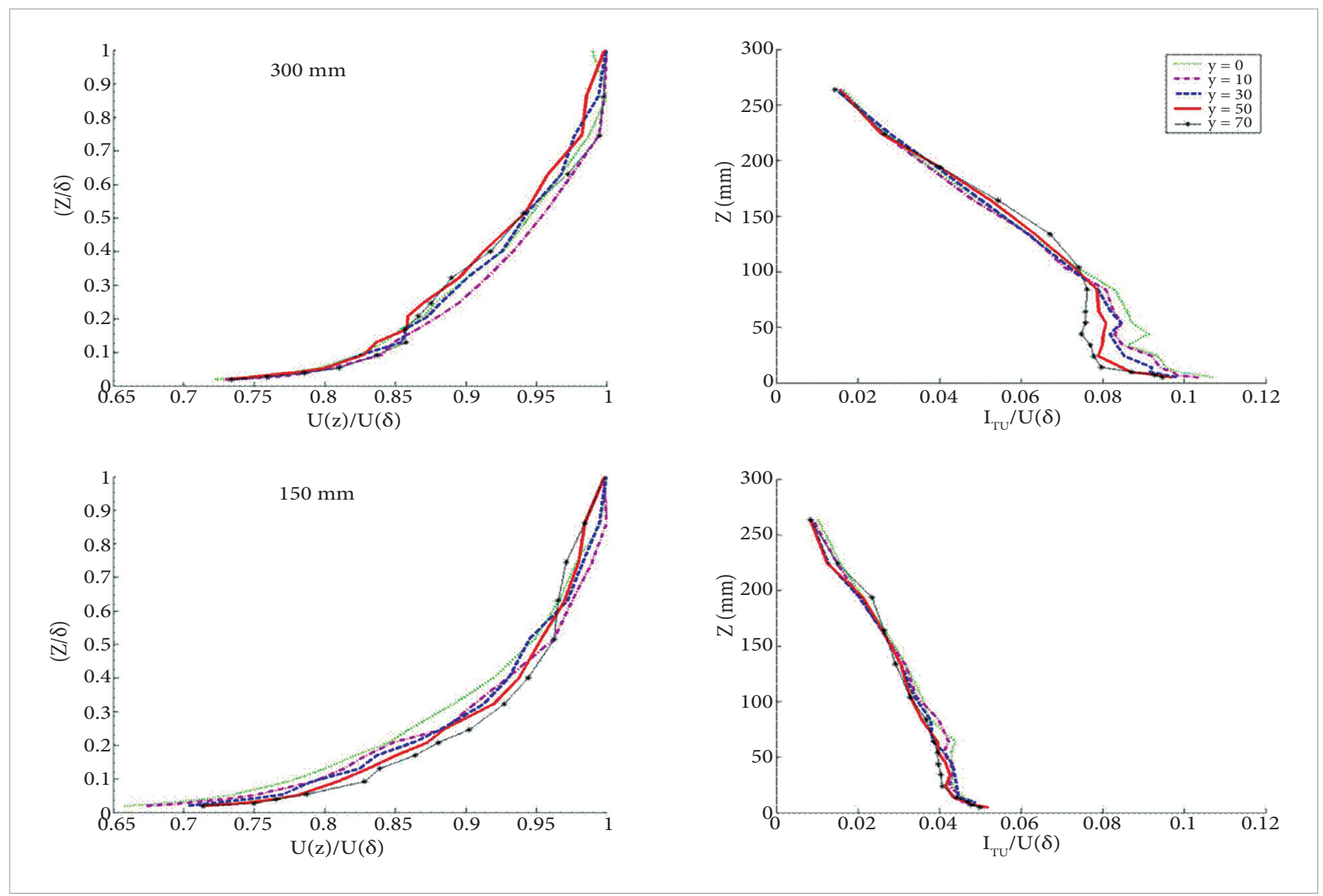

Figure 6. Nondimensional windspeed and turbulence profile at $x=1420 \mathrm{~mm}$. Cases with medium screen (see Table 2). The turbulence level is normalized with respect to the velocity at top of the boundary layer. 
Figure 7 shows the windspeed profile for a medium screen positioned at $150 \mathrm{~mm}$ (named as near) and $300 \mathrm{~mm}$ (far) from the spires, with and without the carpet for fine adjustment. Note that the drag is higher with the carpet, and the $\mathrm{ABL}$ is better formed with the carpet and a medium screen positioned at a $300 \mathrm{~mm}$ distance from the spires. Also, for spires further away from the screen (Fig. 7a), the velocities are greater for low heights, 15 and $25 \mathrm{~m} / \mathrm{s}$, respectively.

Figure 8 shows that when the carpet is used in conjunction with screens and spires, the windspeed profiles become more convex, with the greatest convexity noted for the smallest separation between the spires and screens (150 mm).
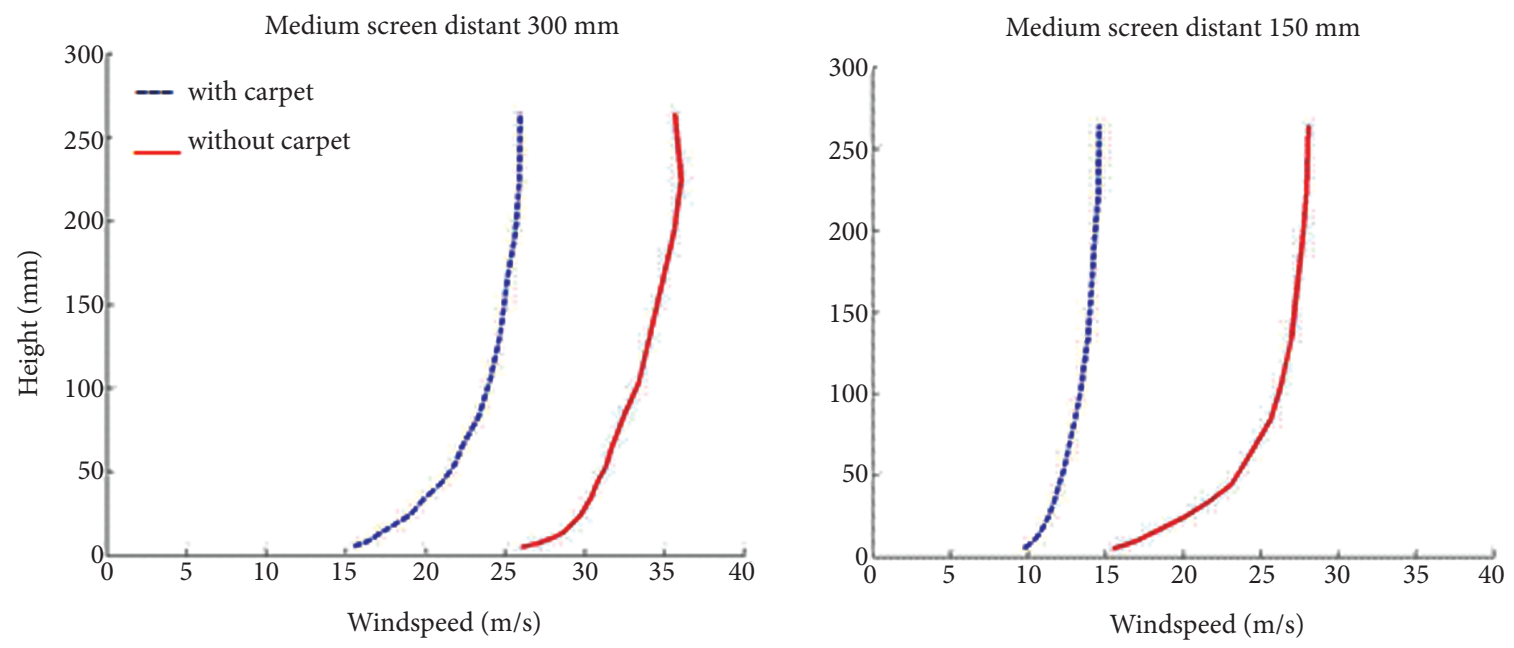

Figure 7. Windspeed profile at $x=1420 \mathrm{~mm}$.
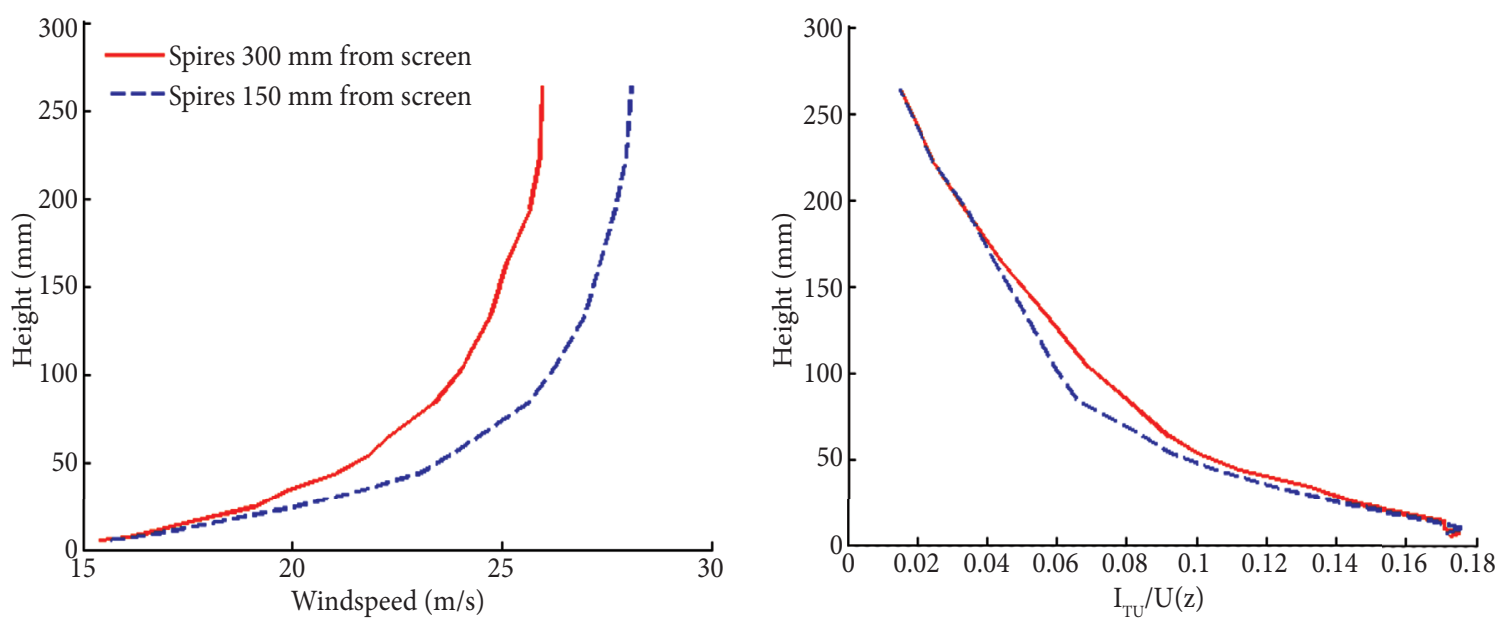

Figure 8. Windspeed profile and turbulent intensity at the point $x=1420 \mathrm{~mm}$ with medium screen and carpet. 
Table 3. Values of $\mathrm{Re}$ and $\alpha$ for tests with the carpet.

\begin{tabular}{|c|c|c|c|c|c|}
\hline Devices & Positions $(\mathrm{mm})$ & Windspeed $(\mathrm{m} / \mathrm{s})$ & Alpha & Re & Height of ABL (mm) \\
\hline Without carpet & $\mathrm{x}=1420$ & 28 & 0.05 & $4.9 \times 10^{5}$ & - \\
\hline Without carpet, with spires close to the screen & $\mathrm{x}=1420$ & 24 & 0.11 & $4.2 \times 10^{5}$ & - \\
\hline With carpet & $\mathrm{x}=1420$ & 25.5 & 0.14 & $4.4 \times 10^{5}$ & 200 \\
\hline With carpet, with spires close to the screen & $\mathrm{x}=1420$ & 25.5 & 0.12 & $4.4 \times 10^{5}$ & 180 \\
\hline
\end{tabular}

Thus, the height of the ABL depends on the distance separating the devices. Adjusting the data to the theoretical power law profile, with $\alpha=0.15$, with the spires $300 \mathrm{~mm}$ away from the screen, an ABL around $200 \mathrm{~mm}$ was obtained, while for the spires closer to the screen (distance of $150 \mathrm{~mm}$ ), the result was an $\mathrm{ABL}$ of $180 \mathrm{~mm}$. The intensity of turbulence showed nonsignificant differences between the two cases.

Table 3 summarizes that Re is not sensitive to the inclusion (or not) of the carpet, varying from $4.2 \times 10^{5}$ to $4.9 \times 10^{5}$. However, the inclusion of the carpet resulted in values of $\alpha$ closer to the ideal 0.15. The height of the ABL increased from 180 to $200 \mathrm{~mm}$ as the distance between the spires and the medium screen increased (from 150 to $300 \mathrm{~mm}$ ). Thus, the mean flow characteristics were apparently met with the combination of spires, medium screen, and carpet. In order to evaluate the results with respect to real ABLs, the mean flow profile was compared to theoretical power law and log-law profiles (Fig. 9). This last one is given by the relation $U(z) / u_{*}=1 / 0.4 \log \left[\left(z-z_{d}\right) / z_{0}\right]$ (Blessman, 1973), where $u_{\star}$ is the friction velocity, $z_{0}$ is the roughness factor, and $z_{d}$ is the zero-plane displacement for very rough surface. Here $z_{d}$ is

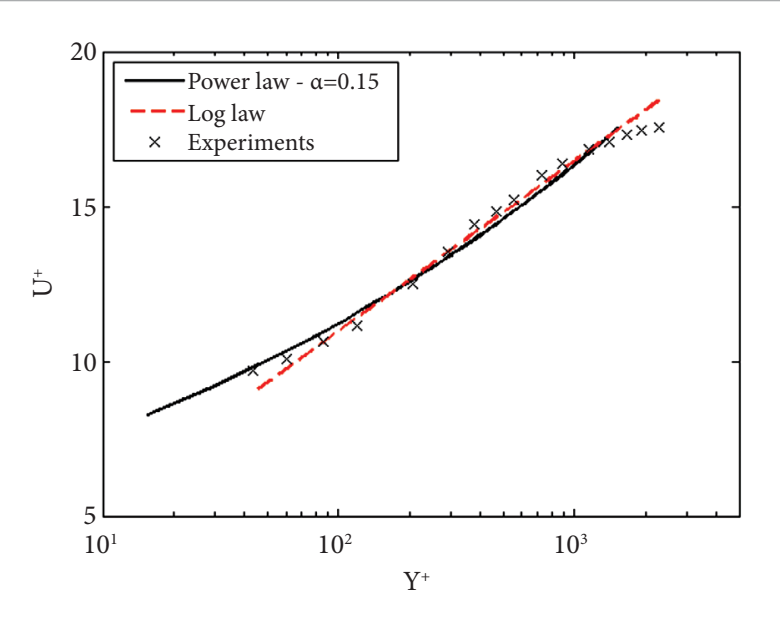

Figure 9. Windspeed profile in comparison to theoretical power law $(\alpha=0.15)$ and log law profiles. assumed to be zero due to the flat terrain conditions and $u_{\star}$ and $z_{0}$ were obtained using the Clauser method. The results of Fig. 9 show a good agreement of the experimental data with the theory. This confirms a good modeling of the mean flow from the ABL at the wind tunnel.

The turbulence intensity is also an important feature of the ABL; therefore its intensities measured in the current experiments were compared to a benchmark suggested by ESDU (Engineering Science Data Unit). Such a benchmark consist of an empirical correlation based on a large experimental database of ABLs measured at height up to $100 \mathrm{~m}$. The expression for this correlation can be found in ESDU (1985) and Liu et al., (2003). Therefore, the current data had to be scaled to the real ABL in order to allow a proper comparison. The results in Fig. 10 show a good agreement between the correlation and the experiments, especially for the streamwise stations where the BL was fully developed ( $\mathrm{x}=1420$ and 1520). At these stations, all experimental results lie between the limit of $\pm 15 \%$, represented in the figure

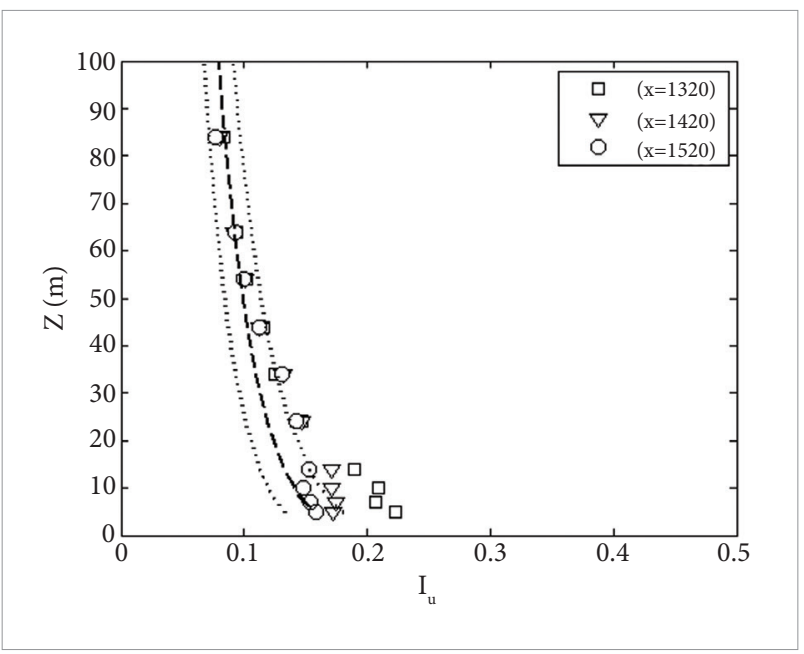

Figure 10. Turbulent intensity at the first $100 \mathrm{~m}$ (equivalent to the boundary layer scaling) and comparison with the correlation suggested by ESDU 7430 and 7431 (Engineering Science Data Unit). The dotted lines represent the $\pm 15 \%$ deviation from the correlation. 


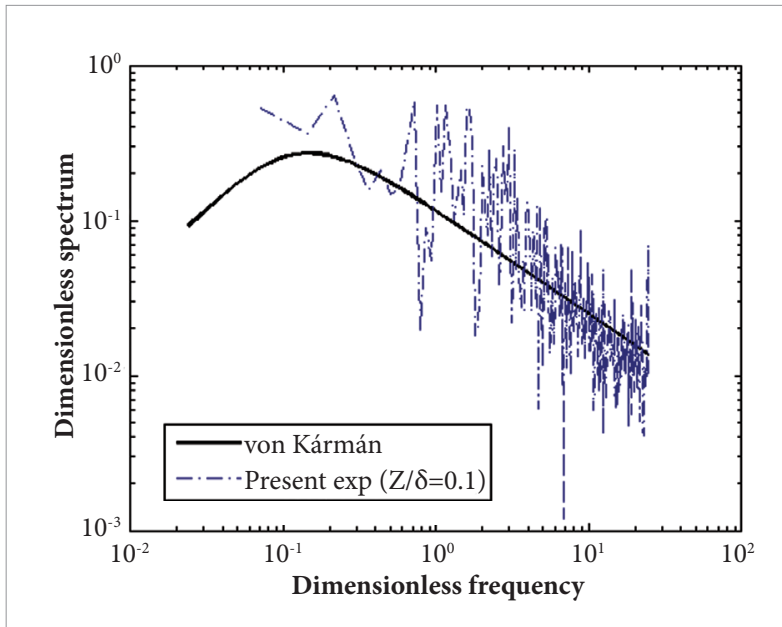

Figure 11. Comparison of the dimensionless spectrum obtained at $\mathrm{z} / \delta=0.1$ and the von Kármán spectrum.

by dotted lines. This suggests that the characteristics of the BL turbulence were also well modeled at the wind tunnel.

In order to complete the assessment of wind tunnel data, the spectrum of flow fluctuations was compared with the vonKármán spectrum (ESDU, 1985), and the results are displayed in Fig. 11. According to ESDU, von-Kármán spectrum is a good model to represent the main features of the longitudinal component of turbulence in ABL. The formula of the dimensionless spectrum is $f S_{u} / \sigma^{2}=4 X_{u}(z) /\left[1+70,78 X_{u}(z)^{2}\right]^{5 / 6}$, where $S_{u}$ is the spectral density function, $f$ the dimensional frequency, $\sigma^{2}$ is the variance, and $X_{u}$ is the dimensionless frequency. The last term is given by $f L(z) / U(z), L(z)$ being the integral scale calculated using the autocorrelation spectra.

A qualitatively good agreement is found between the experiments and the von Kármán spectrum. The region of constant slope that is equivalent to the $-5 / 3$ exponential decay in the inertial subrange of Kolmogorov's cascade is nearly the same in both cases. Unfortunately, a more quantitative comparison could not be made with the current data because the period set for data acquisition was not long enough to enable the use of averaging procedures. Nevertheless, it is clearly observable that the main features of both spectra are the same. Therefore, it is reasonable to assume that mean flow and the turbulence characteristics of the ABL, expected to occur at the coast close to ALC, could be well simulated in a short wind tunnel with the use of passive devices. It is important to point out that for other terrain conditions, especially those at urban regions, this might not be the case.

\section{CONCLUSION}

The ideal situation is the formation of the ABL in the shortest possible extension of the wind tunnel, with the lowest pressure loss possible, to obtain a large Re, similar to the values between $10^{6}$ and $10^{7}$ observed in nature. An ABL can be formed by combinations of passive devices, which do not require a long test section. So, in some situations, existing aeronautical wind tunnels can be used for atmospheric simulations, in particular those with small values of the coefficient $\alpha$ in the power law.

The lowest pressure loss was obtained with the coarse screen alone, while spires followed by a thin screen resulted in the highest pressure loss. The medium screen generated a well-developed $\mathrm{ABL}$ at the mesh point $\mathrm{x}=1420 \mathrm{~mm}$, while the use of thin screen moved the ABL to the position $\mathrm{x}=1120 \mathrm{~mm}$. In view of these findings, if the length of the wind tunnel is critical (being short) and the pressure loss is not so important; the best choice would be the thin screen.

The distance between spires and screen was the other factor examined. A lower pressure loss, with a larger Re equal to $4.8 \times 10^{5}$, was observed with the medium screen more distant from the spires, while for the thin screen Re was equal to $3.9 \times 10^{5}$. However, higher values of $\alpha$ (equal to or greater than 0.10 ) were found for all cases of spires closer to the screens.

The insertion of a carpet caused a sharp increase of $\alpha$, which became closer to the desired value of 0.15 . The ratio of pressure loss between the distances of the spires and the screen remained the same, but demonstrated a smaller drop with the increase of the distance between the devices; however, for the same screen, the height of the ABL was increased with a smaller distance between the screens and spires, resulting in $180 \mathrm{~mm}$ for the distance of $150 \mathrm{~mm}$, and $200 \mathrm{~mm}$ for $300 \mathrm{~mm}$.

These findings indicate that this technique is a suitable tool for generation of an ABL in short-chamber wind tunnel experiments. The main characteristics of wind in an $\mathrm{ABL}$ seem to be well reproduced in the current experiments. This is confirmed by comparisons with 
established benchmarks from the literature. However, an important caveat is that the technique employed here seems to be limited to the reproduction of ABLs of nearly flat terrains, which have very small values of power law exponent. For regions with higher terrain roughness, such as forests and urban areas, the ABL wind tunnels clearly are the most suitable tools.

\section{ACKNOWLEDGMENTS}

Authors acknowledge a doctoral fellowship (CNPq 141861/2006-1) and a Research Scholarship 302117/2004-0, both granted by CNPq (Conselho Nacional de Desenvolvimento Científico e Tecnológico, Brazil).

\section{REFERENCES}

Avelar, A.C., Brasileiro, F., Marto, A.G., Marciotto, E. and Fisch, G., 2012, "Wind Tunnel Simulation of the Atmospheric Boundary Layer for Study of the Wind Pattern at the Alcantara Space Center", Journal of Aerospace and Technology Management, Vol. 4, No. 4, doi: 10.5028/jatm.2012.04044912.

Blessmann, J., 1973, "Simulation of the Natural Wind Structure in an Aerodynamic Wind Tunnel" (in Portuguese), Ph.D. thesis, Instituto Tecnológico de Aeronáutica (ITA), São José dos Campos, S.P., Brazil, 169 p.

Cao, S. and Tamura, T., 2006, "Experimental Study on Roughness Effects on Turbulent Boundary Layer Flow over a Two-Dimensional Steep Hill", Journal of Wind Engineering and Industrial Aerodynamics, Vol. 94, pp. 1-19. doi: 10.1016/j.jweia.2005.10.001.

Carpentieri, M., Hayden, P. and Robins, A.G., 2012, "Wind Tunnel Measurements of Pollutant Turbulent Fluxes in Urban Intersections", Atmospheric Environment, Vol. 46, pp. 669-674. doi: 10.1016/j. atmosenv.2011.09.083.

Davenport, A.G., 1967, "The Dependence of Wind Loads on Meteorological Parameters", Proceedings of the International Seminar on Wind Effects on Buildings and Structures, Ottawa, pp. 19-82.

ESDU, 1985, "Characteristics of atmospheric turbulence near the ground. Part II: Single point data for strong winds (neutral atmosphere)", ESDU International, Item No. 85020, London.

Franck, N., 1963, "Model law and Experimental Technique for Determination of Wind Loads on Buildings", in Symposium on Wind Effects on Buildings and Structures, Vol. 16, National Physical Laboratory, Teddington, UK, pp. 181-196.

Irwin, H.P.A.H., 1981, "The Design of Spires for Wind Simulation", Journal of Wind Engineering and Industrial Aerodynamics, Vol. 7, pp. 361-366, doi: 10.1016/0167-6105(81)90058-1.

Jensen, M. and Franck, N., 1963, "Model Scale Tests in Turbulent Wind", Part I, Danish Technical Press, Copenhagen.

Jensen, M. and Franck, N., 1965, "Model Scale Tests in Turbulent Wind", Part II, Danish Technical Press, Copenhagen.

Kozmar, H., 2009, "Scale Effects in Wind Tunnel Modeling of an Urban Atmospheric Boundary Layer", Theoretical and Applied Climatology, Vol. 100, pp. 153-162, doi: 10.1007/s00704-009-0156-3.
Kwon, K.J., Lee, J.Y. and Sung, B., 2003, "PIV Measurements on the Boundary Layer Flow around Naro Space Center", Proceedings of the 5th International Symposium on Particle Image Velocimetry, pp. 22-24.

Liu, G., Xuan, J. and Park, S., 2003, "A new method to calculate wind profile parameters of the wind tunnel boundary layer", Journal of Wind Engineering and Industrial Aerodynamics, Vol. 91, pp. 1155-1162.

Mavroidis, I., Griffiths, R.F. and Hall, D.J.H., 2003, "Field and Wind Tunnel Investigations of Plume Dispersion around Single Surface Obstacles". Atmospheric Environment, Vol. 37, pp. 2903-2918, doi: 10.1016/S1352-2310(03)00300-5.

Novak, M.D., Warland, J.S., Orchansky, A.L., Ketler, R. and Green, S., 2000, "Wind Tunnel and Field Measurements of Turbulent Flow in Forests. Part I: Uniformly Thinned Stands", Boundary Layer Meteorology, Vol. 95, pp. 457-495, doi: 10.1023/A:1002693625637.

Pires, L.B.M., 2009, "Study of the Internal Boundary Layer Developed on Coastal Cliffs with Application to the Alcântara Launching Center, Brazil" (in Portuguese), Ph.D. thesis, Instituto Nacional de Pesquisas Espaciais (INPE), São José dos Campos, SP, Brazil. 165p, (INPE-16566-TDI/1562), http://pct.capes.gov.br/teses /2009/33010013003P8/TES.PDF.

Pires, L.B.M., Souza, L.F., Fisch, G. and Gielow, R., 2009, "La Influencia de la Altura de la Capa Límite Oceánica en la Región del Centro de Lanzamientos de Alcántara en Brasil”, Información Tecnológica, Vol. 20, pp. 119-128, doi: 10.1612/inf.tecnol.4071it.08.

Pires, L.B.M., Roballo, S.T., Fisch, G., Avelar, A.C., Girardi, R.M. and Gielow, R., 2010, "Atmospheric Flow Measurements Using the PIV and HWA Techniques", Journal of Aerospace Technology and Management, v.2, pp.127-136, doi:10.5028/ jatm.2010.02027410.

Roballo, S.T., 2007, "Estudo do Escoamento Atmosférico na CLA, através de Medidas em Torre Anemométrica e em Túnel de Vento" (in Portuguese), Master thesis, Instituto Nacional de Pesquisas Espaciais (INPE), São José dos Campos, SP, Brazil, 137 p, (INPE14824-TDI/1264).http://mtc-m17.sid.inpe.br/col/sid.inpe.br / mtc-m17@80/2007/06.12.18.07/doc/publicacao.pdf. 